\title{
Examining the attitudes of physical education teachers towards special education (the handicapped)
}

\author{
Yaman Ç. ${ }^{\text {ABCDE }}$, Uluışık V. ${ }^{2 A B C D E}$, Hergüner G. ${ }^{\text {AACDE }}$, Önal A..$^{2 A B C D}$ \\ ${ }^{1}$ Faculty of Sport Science, Recreation, Sakarya University, Turkey \\ ${ }^{2}$ Institute of Educational Science, Physical education and Sport Teaching, Sakarya University, Turkey \\ ${ }^{3}$ Faculty of Sport Science, Physical Education and Sport Teaching, Sakarya University, Turkey
}

Authors' Contribution: A - Study design; B - Data collection; C - Statistical analysis; D - Manuscript Preparation; E - Funds Collection.

\begin{abstract}
Purpose: $\quad$ The purpose of the present study was to determine the education of physical education teachers working at special education schools or classes in this field and to determine the attitudes of them towards students who need special education.

Material: $\quad$ Our study was designed in the form of review model, and 164 questionnaires were included in the analyses. The questionnaires were filled fully by teachers who were selected with the Random Sampling Method and who were contacted in person. The data collection tool used consisted of two parts; Personal Information Form and the Attitude Scale for the Handicapped. Non-parametric tests like Mann Whitney U-Test and Kruskal Wallis H-Test were used in the study because the data did not show normal distribution.

Results: According to the findings, it was determined that the attitudes of the participants in the family life sub-dimension were moderate; and the scores were high in educational medium, interpersonal relations, working life, personal characteristics, competenceindependent life and total attitudes.

Conclusions: As a result, there is a significant loss of productivity due to the lack of special training in physical education teachers who are appointed to special education schools and to job training centers through centralization method.

Keywords: $\quad$ special education, attitude, physical education, sports, teacher, student.
\end{abstract}

\section{Introduction}

Attitudes are not acquired at birth. They are acquired later in life through learning along with social environment in which the interaction takes place in the culture where individual lives. As a social environment, parents, friends, mass media, and personal experiences play a role in the formation of attitudes. It is possible that the attitudes we gain through learning can change [1]. Education and information are effective methods for the society to change their attitudes positively towards the handicapped [2]. Relationship between attitude and behavior is influenced by past experiences and new knowledge [3]. People involved in physical education and sports need to develop positive attitudes toward the handicapped because it is necessary for physical education teachers and for those who will train in this area to have some fieldrelated qualifications for the success of the programs and for the adaptation of the handicapped to physical activity environments. Well-known attitude hypotheses in social psychology may be benefited to explain how the attitudes towards the handicapped are formed and how they can be changed. These hypotheses can help to explain both the formation and the change of attitudes. At the top of these hypotheses, the Learning Approach ranks the first

(c) Yaman Ç., Uluışık V., Hergüner G., Önal A., 2018 doi:10.15561/20755279.2018.0406
$[4,5,6]$. In addition, many techniques are used to change the negative attitudes towards the handicapped. The most commonly used techniques are informing, simulating and establishing personal relationships.

Education is a long-term process and there are many factors that affect this process. Everybody in training knows that there are individual differences in education. These differences are sometimes so deep that some of the students' social, emotional, physical, and cognitive competences and developments in the teaching process do not proceed at the same level as their peers. Children who need additional support services due to their social, emotional, physical and cognitive difficulties also need additional resources to benefit from the training they receive [7]. These sources are daily instructional processes and teacher-student relations and other factors shaping the classroom setting [8]. With a clearer statement, the needs of students who need special education can be met with classes that address them, with special education materials, and with teachers who are trained in that area.

Teachers responsible for education and the relevant departments of the universities need to act and cooperate with each other to work on enhancing the quality of teachers in order to develop a comprehensive and collaborative approach to the qualifications of the private 
educators, to fully discuss the teacher competencies in the special education and to develop comprehensive reforms to eliminate these problems $[9,10]$.

It is known that branch teachers working in special education schools and classes can be assigned to these schools without having undergraduate education on special education. One of these branches is physical education. Physical education teachers, like other branch teachers who work in special education schools, also experience difficulties. Some of physical education teachers working in these schools have not received any training in special education at the undergraduate level, or they continue to work in these institutions by taking courses for two or four credits only for one semester. For this reason, teachers assigned to special education institutions face difficulties both for themselves and for their students.

Previous studies show that if people are conscious about special education, their attitudes towards individuals who need special education will experience a positive development. This study was carried out in order to determine the education of the physical education teachers working in the special education schools or classes; and the attitudes of the physical education teachers to the individuals who need special education. At the end of the study, it will be possible to enlighten the scientific relevance of the attitudes of physical education teachers towards the education levels of special education and the individuals who need special education.

\section{Material and Methods}

This study was designed in the screening model to determine the attitudes of physical education teachers towards students who need special education and to determine how physical education teachers are trained for students with special education needs. In this context, in our examinations on the programs at universities, we determined that as of 2017, we can see that the special education course exists in the undergraduate programs of physical education and sports teachers as 2 credits in the $4^{\text {th }}$ semester and physical education and sports for the handicapped exists in the $7^{\text {th }}$ semester a 2 credit-classes in the curriculum of the Higher Education Council (HEC).

When we examine the undergraduate programs of Ege University, Manisa Celal Bayar University, Marmara University, Gazi University and Sakarya University Sport Science Faculties, which we determined by the Random Sampling Method, we see that there are different practices.

For example, Gazi University, Ege University, Marmara University and Manisa Celal Bayar University, although some of which have them in different periods, special education and physical education and sports classes for the handicapped students are included in the compulsory courses. When we look at Sakarya University, Sports Sciences Faculty, Physical Education and Sports Teaching Program, we can see that these courses are not included in the compulsory or elective courses. Taking these differences into consideration, this study was carried out in order to determine the competence of today's physical education and sport teaching programs in terms of special education and the quantity and quality of previous physical education and sport teacher education programs.

\section{Participants}

The sample group of our study consisted of the participants who worked at physical education teachers' departments of schools of the Ministry of Education in various areas of Turkey. Our survey was applied to 180 physical education teachers who were selected and reached by Random Sampling Method. 21 of the questionnaire forms that were collected were excluded from the study because of having incomplete data or being filled mistakenly, and 164 questionnaire forms were included in the study.

\section{Procedure}

The questionnaires were used to collect the data. The questionnaire form applied to physical education teachers consisted of two parts; Demographic Information and Attitude Scale towards the Handicapped.

\section{The Attitude Scale towards the Handicapped}

The scale was prepared by the Project Research Team of the Optimar Research Company in order to evaluate the social attitudes towards disability by the Prime Minister's Office, Administration for Disabled People [11]. There are a total of 43 statements in the scale, which consists of 6 sub-dimensions.

The Cronbach Alfa Internal Consistency Coefficients of the Attitude Scale towards the Handicapped were computed as; Educational Medium - EM 0.54; Interpersonal Relations - IR 0.74; Work Life - WL 0.71; Family Life - FL 0.61; Personal Characteristics - PC 0.75; Competence - Independent Life - CIL 0.82; for the whole test as 0.88 .

As shown above, the Cronbach Alpha Internal Consistency Coefficients of the subscales and that of the scale vary between 0.54 and 0.88 . Therefore, it is accepted that the scale is a reliable measurement tool.

\section{Statistical Analysis}

The SPSS 22.0 Program was used for the analysis of the obtained data. Before comparing the scale scores according to the demographic information of the participants, it was examined whether the scale scores showed normal distribution or not. It was determined in the normality test that the scale subscales and total scores did not show normal distribution. For this reason, nonparametric tests were used to compare the scale scores according to demographic information of the participants.

The Mann Whitney U-test was used to compare the scale scores according to the gender of the participants, their level of education and whether they wanted to work in a school that provided special education. The Kruskal Wallis H-test was used for the comparison of the scale scores according to age groups, graduated departments, receiving special education status, working status in a special education institution and whether they found themselves competent about special education. 


\section{Results}

When the table is examined, it is seen that the scores of the participants are high in Family Life subdimension, and their scores are high in Educational Medium, Interpersonal Relations, Work Life, Personal Characteristics, Competence - Independent Life and Total Attitude.

When the table is examined it is seen that the attitudes of the participants towards the handicapped do not differ according to the gender variable in sub-dimension and in general attitude levels at a statistically significant level $(\mathrm{p}>0,05)$.

When the table is examined, it is seen that the attitudes of the participants towards the handicapped do not differ at a statistically significant level in terms of age groups in sub-dimensions and in general attitude levels ( $p>0,05)$.

Table 1. Frequency Distributions on the Demographical Data of the Participants

\begin{tabular}{|c|c|c|c|}
\hline Variables & Sub-variables & $\mathbf{F}$ & $\%$ \\
\hline \multirow{3}{*}{ Gender } & Female & 62 & 37,8 \\
\hline & Male & 102 & 62,2 \\
\hline & $22-30$ age & 2 & 1,2 \\
\hline \multirow{3}{*}{ Age groups } & 31-40 age & 77 & 47,0 \\
\hline & $41-50$ age & 78 & 47,6 \\
\hline & $50+$ age & 7 & 4,3 \\
\hline \multirow{3}{*}{ Educational Status } & Undergraduate & 130 & 79,3 \\
\hline & Postgraduate & 34 & 20,7 \\
\hline & Physical education teaching field & 152 & 92,7 \\
\hline \multirow[t]{3}{*}{ Department graduated } & Class Teachers Department & 9 & 5,5 \\
\hline & Sports man. tra. Ad. & 3 & 1,8 \\
\hline & I did not receive education & 108 & 65,9 \\
\hline \multirow{2}{*}{$\begin{array}{l}\text { Receiving special education } \\
\text { status }\end{array}$} & $\begin{array}{l}\text { I received phy. ed. and sports for the Handicapped } \\
\text { classes }\end{array}$ & 39 & 23,8 \\
\hline & $\begin{array}{l}\text { I received course and seminars about the } \\
\text { handicapped }\end{array}$ & 17 & 10,4 \\
\hline \multirow{3}{*}{$\begin{array}{l}\text { Working status at a school } \\
\text { providing special education }\end{array}$} & I did not work & 134 & 81,7 \\
\hline & I worked & 24 & 14,6 \\
\hline & I still work & 6 & 3,7 \\
\hline \multirow{3}{*}{$\begin{array}{l}\text { Feeling competent in the } \\
\text { field of special education }\end{array}$} & No & 72 & 43,9 \\
\hline & Partly & 78 & 47,6 \\
\hline & Yes & 14 & 8,5 \\
\hline \multirow{2}{*}{$\begin{array}{l}\text { The desire for working at a } \\
\text { school that provides special } \\
\text { education }\end{array}$} & Yes & & 44,5 \\
\hline & No & 91 & 55,5 \\
\hline
\end{tabular}

Table 2. Definitive Statistics on the Attitude Levels of the Participants towards the Handicapped

\begin{tabular}{lll}
\hline Sub-dimensions & $\mathbf{X}$ & Sd \\
\hline Educational Medium & 13,62 & 1,674 \\
Interpersonal Relations & 37,80 & 4,763 \\
Work Life & 37,16 & 5,131 \\
Family Life & 10,00 & 2,381 \\
Personal Characteristics & 28,44 & 3,845 \\
Competence - Independent Life & 49,22 & 6,330 \\
Total Attitude Score & 176,25 & 20,483 \\
\hline
\end{tabular}

Table 3. Comparison of Attitude Levels of the Participants according to Gender Variable

\begin{tabular}{|c|c|c|c|c|c|c|c|c|}
\hline Sub-dimensions & Gender & $\mathbf{N}$ & $x$ & Sd & $\begin{array}{l}\text { Rank } \\
\text { Ave. }\end{array}$ & Rank total & $\mathbf{U}$ & $\mathbf{p}$ \\
\hline \multirow{2}{*}{ Educational Medium } & Female & 62 & 13,48 & 1,627 & 76,15 & 4721,0 & \multirow{2}{*}{2768,0} & \multirow{2}{*}{ 161 } \\
\hline & Male & 102 & 13,71 & 1,704 & 86,36 & 8809,0 & & \\
\hline \multirow{2}{*}{$\begin{array}{l}\text { Interpersonal } \\
\text { Relations }\end{array}$} & Female & 62 & 37,45 & 4,738 & 77,93 & 4831,5 & \multirow{2}{*}{2878,5} & \multirow{2}{*}{,335 } \\
\hline & Male & 102 & 38,02 & 4,789 & 85,28 & 8698,5 & & \\
\hline \multirow{2}{*}{ Work Life } & Female & 62 & 36,76 & 5,059 & 78,58 & 4872,0 & \multirow{2}{*}{2919,0} & \multirow{2}{*}{,409 } \\
\hline & Male & 102 & 37,41 & 5,183 & 84,88 & 8658,0 & & \\
\hline \multirow{2}{*}{ Family Life } & Female & 62 & 10,15 & 2,604 & 85,81 & 5320,5 & \multirow{2}{*}{2956,5} & \multirow{2}{*}{,482 } \\
\hline & Male & 102 & 9,91 & 2,243 & 80,49 & 8209,5 & & \\
\hline \multirow{3}{*}{$\begin{array}{l}\text { Personal } \\
\text { Characteristics } \\
\text { Competence - }\end{array}$} & Female & 62 & 28,34 & 3,862 & 81,04 & 5024,5 & \multirow{2}{*}{3071,5} & \multirow{2}{*}{,758 } \\
\hline & Male & 102 & 28,50 & 3,853 & 83,39 & 8505,5 & & \\
\hline & Female & 62 & 49,00 & 6,445 & 79,46 & 4926,5 & \multirow{2}{*}{2973,5} & \multirow{2}{*}{, 522} \\
\hline Independent Life & Male & 102 & 49,35 & 6,288 & 84,35 & 8603,5 & & \\
\hline \multirow{2}{*}{ Total Attitude Score } & Female & 62 & 175,18 & 21,051 & 79,19 & 4910,0 & \multirow{2}{*}{2957,0} & \multirow{2}{*}{,487 } \\
\hline & Male & 102 & 176,90 & 20,207 & 84,51 & 8620,0 & & \\
\hline
\end{tabular}


Table 4. Comparison of the Attitude towards the Handicapped Scores of the Participants according to the Age Groups

\begin{tabular}{|c|c|c|c|c|c|c|c|}
\hline Sub-dimensions & Age Groups & $\mathbf{N}$ & $\mathrm{X}$ & Sd & Rank Ave. & $x^{2}$ & p \\
\hline \multirow{4}{*}{ Educational Medium } & 22-30 Age & 2 & 12,00 & 4,243 & 68,00 & \multirow{4}{*}{5,144} & \multirow{4}{*}{ 162 } \\
\hline & 31-40 Age & 77 & 13,81 & 1,442 & 84,69 & & \\
\hline & 41-50 Age & 78 & 13,38 & 1,832 & 77,69 & & \\
\hline & $50+$ Age & 7 & 14,71 & ,488 & 116,21 & & \\
\hline \multirow{3}{*}{ Interpersonal Relations } & 22-30 Age & 2 & 37,50 & 7,778 & 73,25 & \multirow{4}{*}{,420 } & \multirow{4}{*}{,936 } \\
\hline & $\begin{array}{l}\text { 31-40 Age } \\
\text { 41-50 Age }\end{array}$ & $\begin{array}{l}77 \\
78\end{array}$ & $\begin{array}{l}38,13 \\
3746\end{array}$ & $\begin{array}{l}4,134 \\
5,453\end{array}$ & $\begin{array}{l}84,38 \\
80,44\end{array}$ & & \\
\hline & $50+$ Age & 7 & 38,14 & 2,268 & 87,43 & & \\
\hline \multirow{4}{*}{ Work Life } & 22-30 Age & 2 & 34,00 & 5,657 & 54,50 & & \\
\hline & 31-40 Age & 77 & 37,64 & 5,119 & 87,47 & \multirow{3}{*}{2,274} & \multirow{4}{*}{,518 } \\
\hline & 41-50 Age & 78 & 36,77 & 5,045 & 78,03 & & \\
\hline & $50+$ Age & 7 & 37,29 & 6,550 & 85,64 & & \\
\hline \multirow{4}{*}{ Family Life } & 22-30 Age & 2 & 11,00 & 1,414 & 105,00 & \multirow{4}{*}{4,640} & \\
\hline & 31-40 Age & 77 & 9,97 & 2,449 & 81,44 & & \multirow{3}{*}{ 200 } \\
\hline & 41-50 Age & 78 & 10,18 & 2,266 & 86,04 & & \\
\hline & $50+$ Age & 7 & 8,00 & 2,517 & 48,29 & & \\
\hline \multirow{4}{*}{ Personal Characteristics } & 22-30 Age & 2 & 28,00 & 5,657 & 74,75 & \multirow{4}{*}{,435 } & \\
\hline & 31-40 Age & 77 & 28,65 & 4,048 & 84,49 & & \multirow{3}{*}{,933 } \\
\hline & 41-50 Age & 78 & 28,21 & 3,683 & 80,29 & & \\
\hline & 50+ Age & 7 & 28,86 & 3,625 & 87,43 & & \\
\hline \multirow{3}{*}{$\begin{array}{l}\text { Competence - } \\
\text { Independent Life }\end{array}$} & 22-30 Age & 2 & 44,00 & 7,071 & 42,00 & \multirow{4}{*}{2,580} & \multirow{4}{*}{,461 } \\
\hline & 31-40 Age & 77 & 49,99 & 5,576 & 86,82 & & \\
\hline & $\begin{array}{l}41-50 \text { Age } \\
50+\text { Age }\end{array}$ & 78 & $\begin{array}{l}48,65 \\
4857\end{array}$ & $\begin{array}{l}7,067 \\
4,928\end{array}$ & 80,13 & & \\
\hline \multirow{4}{*}{ Total Attitude Score } & 22-30 Age & 2 & 166,50 & 31,820 & 66,50 & & \\
\hline & 31-40 Age & 77 & 178,18 & 18,965 & 86,03 & \multirow{3}{*}{ 981 } & \multirow{3}{*}{,806 } \\
\hline & 41-50 Age & 78 & 174,65 & 22,104 & 79,42 & & \\
\hline & $50+$ Age & 7 & 175,57 & 17,018 & 82,50 & & \\
\hline
\end{tabular}

Table 5. Comparison of the Attitudes of the Participants towards the Handicapped according to Educational Status

\begin{tabular}{|c|c|c|c|c|c|c|c|c|}
\hline Sub-dimensions & $\begin{array}{l}\text { Educational } \\
\text { Status }\end{array}$ & $\mathbf{N}$ & $\mathbf{x}$ & Sd & $\begin{array}{l}\text { Rank } \\
\text { Ave. }\end{array}$ & Rank Total & $\mathbf{U}$ & $\mathbf{p}$ \\
\hline Educational & Undergraduate & 130 & 13,61 & 1,659 & 81,88 & 10644,0 & \multirow{2}{*}{2129,0} & \multirow[b]{2}{*}{,730 } \\
\hline Medium & Post-graduate & 34 & 13,68 & 1,753 & 84,88 & 2886,0 & & \\
\hline Interpersonal & Undergraduate & 130 & 37,82 & 4,852 & 83,31 & 10830,0 & \multirow{2}{*}{2105,0} & \multirow{2}{*}{,669 } \\
\hline Relations & Post-graduate & 34 & 37,74 & 4,474 & 79,41 & 2700,0 & & \\
\hline Work Life & $\begin{array}{l}\text { Undergraduate } \\
\text { Post-graduate }\end{array}$ & $\begin{array}{l}130 \\
34\end{array}$ & $\begin{array}{l}37,41 \\
36,24\end{array}$ & $\begin{array}{l}4,944 \\
5,774\end{array}$ & $\begin{array}{l}84,51 \\
74,81\end{array}$ & $\begin{array}{l}10986,5 \\
2543,5\end{array}$ & 1948,5 & 288 \\
\hline Family Life & $\begin{array}{l}\text { Undergraduate } \\
\text { Post-graduate }\end{array}$ & $\begin{array}{l}130 \\
34\end{array}$ & $\begin{array}{l}10,20 \\
9,24\end{array}$ & $\begin{array}{l}2,327 \\
2,463\end{array}$ & $\begin{array}{l}86,02 \\
69,03\end{array}$ & $\begin{array}{l}11183,0 \\
2347,0\end{array}$ & 1752,0 & ,061 \\
\hline Personal & Undergraduate & 130 & 28,61 & 3,883 & 84,95 & 11044,0 & \multirow{2}{*}{1891,0} & \multirow{2}{*}{ 194 } \\
\hline Characteristics & Post-graduate & 34 & 27,79 & 3,683 & 73,12 & 2486,0 & & \\
\hline Competence - & Undergraduate & 130 & 49,34 & 6,682 & 84,26 & 10953,5 & \multirow{2}{*}{1981,5} & \multirow{2}{*}{ 353 } \\
\hline Independent Life & Post-graduate & 34 & 48,76 & 4,812 & 75,78 & 2576,5 & & \\
\hline Total Attitude & Undergraduate & 130 & 176,98 & 20,759 & 84,78 & 11021,0 & \multirow{2}{*}{1914,0} & \multirow{2}{*}{ 230 } \\
\hline Score & Post-graduate & 34 & 173,44 & 19,428 & 73,79 & 2509,0 & & \\
\hline
\end{tabular}

Table 6. Comparison of the Attitude towards the Handicapped Scores of the Participants according to the Graduation Fields

\begin{tabular}{|c|c|c|c|c|c|c|c|}
\hline Sub-dimensions & $\begin{array}{l}\text { The department } \\
\text { graduated }\end{array}$ & $\mathbf{N}$ & $\mathbf{X}$ & Sd & Rank Ave. & $x^{2}$ & $\mathbf{p}$ \\
\hline \multirow{3}{*}{$\begin{array}{l}\text { Educational } \\
\text { Medium }\end{array}$} & Phy. Ed. Teach. & 152 & 13,55 & 1,710 & 80,64 & \multirow{3}{*}{4,475} & \multirow{3}{*}{ 107 } \\
\hline & Class Teach. & 9 & 14,67 &, 500 & 113,50 & & \\
\hline & Sports Manag. Tra. & 3 & 14,00 & 1,000 & 83,83 & & \\
\hline \multirow{3}{*}{$\begin{array}{l}\text { Interpersonal } \\
\text { Relations }\end{array}$} & Phy. Ed. Teach. & 152 & 37,67 & 4,813 & 81,11 & \multirow{3}{*}{2,210} & \multirow{3}{*}{ 331 } \\
\hline & Class Teach. & 9 & 39,89 & 3,219 & 105,22 & & \\
\hline & $\begin{array}{l}\text { Sports Manag. Tra. } \\
\text { Phy. Ed. Teach. }\end{array}$ & $\begin{array}{l}3 \\
152\end{array}$ & $\begin{array}{l}38,33 \\
37,09\end{array}$ & $\begin{array}{l}6,110 \\
5,157\end{array}$ & $\begin{array}{l}84,67 \\
81,66\end{array}$ & & \\
\hline \multirow[t]{2}{*}{ Work Life } & Class Teach. & 9 & 39,11 & 3,551 & 101,33 & \multirow[t]{2}{*}{1,732} & \multirow[t]{2}{*}{,421 } \\
\hline & Sports Manag. Tra. & $\begin{array}{l}3 \\
152\end{array}$ & $\begin{array}{l}35,33 \\
10,04\end{array}$ & $\begin{array}{l}8,021 \\
2,392\end{array}$ & $\begin{array}{l}68,50 \\
83,32\end{array}$ & & \\
\hline \multirow[t]{2}{*}{ Family Life } & Class Teach. & 9 & 9,33 & 2,598 & 68,72 & \multirow[t]{2}{*}{,817 } & \multirow[t]{2}{*}{,665 } \\
\hline & Sports Manag. Tra. & 3 & 10,00 & 1,000 & 82,50 & & \\
\hline \multirow{3}{*}{$\begin{array}{l}\text { Personal } \\
\text { Characteristics }\end{array}$} & Phy. Ed. Teach. & 152 & 28,43 & 3,868 & 82,40 & \multirow{3}{*}{1,888} & \multirow{3}{*}{ 389 } \\
\hline & Class Teach. & 9 & 29,33 & 3,873 & 94,56 & & \\
\hline & Sports Manag. Tra. & 3 & 26,00 & 1,732 & 51,33 & & \\
\hline \multirow{2}{*}{$\begin{array}{l}\text { Competence - } \\
\text { Independent Life }\end{array}$} & Phy. Ed. Teach. & 152 & 49,02 & 6,468 & 80,67 & \multirow[b]{2}{*}{3,500} & \multirow[b]{2}{*}{ 174 } \\
\hline & $\begin{array}{l}\text { Class Teach. } \\
\text { Snors }\end{array}$ & 9 & 52,33 & 3,428 & 110,67 & & \\
\hline \multirow{3}{*}{$\begin{array}{l}\text { Total Attitude } \\
\text { Score }\end{array}$} & Phy. Ed. Teach. & 152 & 175,80 & 20,779 & 81,61 & \multirow{3}{*}{1,413} & \multirow{3}{*}{ 493 } \\
\hline & Class Teach. & 9 & 184,67 & 14,722 & 100,33 & & \\
\hline & Sports Manag. Tra. & 3 & 173,67 & 19,604 & 74,33 & & \\
\hline
\end{tabular}


When the table is examined, it is seen that the attitudes of the participants towards the handicapped do not differ at a statistically significant level in terms of educational status in sub-dimensions and in general attitude levels $(\mathrm{p}>0,05)$.

When the table is examined, it is seen that the attitudes of the participants towards the handicapped do not differ at a statistically significant level in terms of the fields graduated in sub-dimensions and in general attitude levels $(\mathrm{p}>0,05)$.

When the table is examined, it is seen that the attitude score levels of the participants do not differ at a statistically significant level in terms of having received education in sub-dimensions and in general attitude levels ( $p>0,05)$.

When the table is examined, it is seen that the attitude levels of the participants do not differ at a statistically

Table 7. Comparison of the Attitudes of the Participants towards the Handicapped according to Having Received Education on Special Education (the Handicapped)

\begin{tabular}{|c|c|c|c|c|c|c|c|}
\hline Sub-dimensions & Educational status & $\mathbf{N}$ & $x$ & Sd & $\begin{array}{l}\text { Rank } \\
\text { Ave. }\end{array}$ & $x^{2}$ & p \\
\hline & I did not receive education & 108 & 13,44 & 1,731 & 76,83 & \multirow{3}{*}{5,176} & \multirow{3}{*}{075} \\
\hline \multirow[t]{3}{*}{$\begin{array}{l}\text { Educational } \\
\text { Medium }\end{array}$} & $\begin{array}{l}\text { I received education on Phy. Ed. \& } \\
\text { Sports for the Handicapped }\end{array}$ & 39 & 13,97 & 1,405 & 91,58 & & \\
\hline & $\begin{array}{l}\text { I received course and seminar on the } \\
\text { Handicapped }\end{array}$ & 17 & 14,00 & 1,768 & 97,71 & & \\
\hline & I did not receive education & 108 & 37,71 & 4,630 & 81,83 & \multirow{3}{*}{ 115 } & \multirow{3}{*}{,944 } \\
\hline \multirow[t]{3}{*}{$\begin{array}{l}\text { Interpersonal } \\
\text { Relations }\end{array}$} & $\begin{array}{l}\text { I received education on Phy. Ed. \& } \\
\text { Sports for the Handicapped }\end{array}$ & 39 & 38,05 & 4,651 & 82,83 & & \\
\hline & $\begin{array}{l}\text { I received course and seminar on the } \\
\text { Handicapped }\end{array}$ & 17 & 37,82 & 6,013 & 85,97 & & \\
\hline & I did not receive education & 108 & 37,01 & 4,877 & 80,45 & \multirow{3}{*}{,785 } & \multirow{3}{*}{675} \\
\hline \multirow[t]{3}{*}{ Work Life } & $\begin{array}{l}\text { I received education on Phy. Ed. \& } \\
\text { Sports for the Handicapped }\end{array}$ & 39 & 37,28 & 5,740 & 84,60 & & \\
\hline & $\begin{array}{l}\text { I received course and seminar on the } \\
\text { Handicapped }\end{array}$ & 17 & 37,88 & 5,487 & 90,68 & & \\
\hline & I did not receive education & 108 & 10,11 & 2,361 & 84,87 & \multirow{3}{*}{2,076} & \multirow{3}{*}{,354 } \\
\hline \multirow[t]{3}{*}{ Family Life } & $\begin{array}{l}\text { I received education on Phy. Ed. \& } \\
\text { Sports for the Handicapped }\end{array}$ & 39 & 10,05 & 2,554 & 82,63 & & \\
\hline & $\begin{array}{l}\text { I received course and seminar on the } \\
\text { Handicapped }\end{array}$ & 17 & 9,18 & 2,038 & 67,18 & & \\
\hline & I did not receive education & 108 & 28,47 & 3,824 & 83,50 & \multirow{3}{*}{, 160 } & \multirow{3}{*}{,923 } \\
\hline \multirow[t]{3}{*}{$\begin{array}{l}\text { Personal } \\
\text { Characteristics }\end{array}$} & $\begin{array}{l}\text { I received education on Phy. Ed. \& } \\
\text { Sports for the Handicapped }\end{array}$ & 39 & 28,36 & 4,101 & 79,99 & & \\
\hline & $\begin{array}{l}\text { I received course and seminar on the } \\
\text { Handicapped }\end{array}$ & 17 & 28,41 & 3,589 & 81,94 & & \\
\hline & I did not receive education & 108 & 48,99 & 6,257 & 80,50 & \multirow{3}{*}{,571 } & \multirow{3}{*}{,752 } \\
\hline \multirow[t]{3}{*}{$\begin{array}{l}\text { Competence - } \\
\text { Independent Life }\end{array}$} & $\begin{array}{l}\text { I received education on Phy. Ed. \& } \\
\text { Sports for the Handicapped }\end{array}$ & 39 & 49,92 & 6,175 & 86,63 & & \\
\hline & $\begin{array}{l}\text { I received course and seminar on the } \\
\text { Handicapped }\end{array}$ & 17 & 49,06 & 7,369 & 85,76 & & \\
\hline & I did not receive education & 108 & 175,73 & 20,101 & 81,84 & \multirow{3}{*}{,066 } & \multirow{3}{*}{,967 } \\
\hline \multirow[t]{2}{*}{$\begin{array}{l}\text { Total Attitude } \\
\text { Score }\end{array}$} & $\begin{array}{l}\text { I received education on Phy. Ed. \& } \\
\text { Sports for the Handicapped }\end{array}$ & 39 & 177,64 & 21,255 & 83,45 & & \\
\hline & $\begin{array}{l}\text { I received course and seminar on the } \\
\text { Handicapped }\end{array}$ & 17 & 176,35 & 22,192 & 84,50 & & \\
\hline
\end{tabular}


significant level in terms of working in a school that provides special education in Sub-dimensions and in general attitude levels ( $\mathrm{p}>0,05)$.

When the table is examined, it is seen that the attitude scores of the participants do not differ at a statistically significant level in terms of feeling competent in special education for the handicapped in sub-dimensions and in general attitude levels ( $\mathrm{p}>0,05)$.

When the table is examined it is seen that the attitude scores of the participants do not differ according to the desire for working in a special education school in subdimensions and in general attitude levels at a statistically significant level $(\mathrm{p}>0,05)$.

\section{Discussion}

When the literature is examined, similar studies were observed although they did not cover the same study topic of ours. When we examine the studies conducted so far, it seems that the techniques of establishing personal relationships, simulation and informing (training in a specific subject) significantly affect the attitudes of people in a certain set of subjects [12]. In the study by Sazak-Pinar [13] conducted on classroom teachers with classroom integration students to determine their inservice training needs, it was stated that more than 50\% of the teachers needed all knowledge or skills except 4 items in data collection tool. The knowledge or skills

Table 8. Comparison of the Attitudes of the Participants towards the Handicapped according to the Working Status in a School that Provides Education on Special Education (the Handicapped)

\begin{tabular}{|c|c|c|c|c|c|c|c|}
\hline Sub-dimensions & Working Status & $\mathbf{N}$ & $\mathbf{x}$ & Sd & $\begin{array}{l}\text { Rank } \\
\text { Ave. }\end{array}$ & $x^{2}$ & $\mathbf{p}$ \\
\hline & I did not work & 134 & 13,57 & 1,753 & 81,96 & & \\
\hline \multirow[t]{3}{*}{ Educational Medium } & I worked & 24 & 13,88 & 1,296 & 85,90 & 160 & 923 \\
\hline & Still at work & 6 & 13,83 & 1,169 & 81,08 & & \\
\hline & I did not work & 134 & 37,85 & 4,553 & 82,74 & & \\
\hline \multirow[t]{3}{*}{ Interpersonal Relations } & I worked & 24 & 37,21 & 6,115 & 77,40 & 885 & 643, \\
\hline & Still at work & 6 & 39,17 & 3,488 & 97,50 & & \\
\hline & I did not work & 134 & 37,22 & 4,994 & 82,82 & & \\
\hline \multirow[t]{3}{*}{ Work Life } & I worked & 24 & 36,50 & 6,345 & 78,44 & 399, & ,819 \\
\hline & Still at work & 6 & 38,50 & 2,258 & 91,50 & & \\
\hline & I did not work & 134 & 9,98 & 2,398 & 82,16 & & \\
\hline \multirow[t]{3}{*}{ Family Life } & I worked & 24 & 10,08 & 2,412 & 83,38 & ,058 & 971 \\
\hline & Still at work & 6 & 10,17 & 2,229 & 86,50 & & \\
\hline & I did not work & 134 & 28,46 & 3,887 & 83,21 & & \\
\hline \multirow[t]{2}{*}{ Personal Characteristics } & I worked & 24 & 28,29 & 3,962 & 77,96 & ,265 & 876 \\
\hline & Still at work & 6 & 28,50 & 2,811 & 84,75 & & \\
\hline \multirow{4}{*}{$\begin{array}{l}\text { Competence - Independent } \\
\text { Life }\end{array}$} & I did not work & 134 & 49,25 & 5,865 & 81,57 & & \\
\hline & I worked & 24 & 48,75 & 9,124 & 84,38 & ,553 & ,759 \\
\hline & Still at work & 6 & 50,50 & 2,345 & 95,67 & & \\
\hline & I did not work & 134 & 176,33 & 19,752 & 82,30 & & \\
\hline \multirow[t]{2}{*}{ Total Attitude Score } & I worked & 24 & 174,71 & 26,462 & 81,23 & ,264 & ,876 \\
\hline & Still at work & 6 & 180,67 & 6,593 & 92,08 & & \\
\hline
\end{tabular}

Table 9. Comparison of the Attitudes of the Participants towards the Handicapped according to the Feeling Competent in Special Education Field (the handicapped)

\begin{tabular}{|c|c|c|c|c|c|c|c|}
\hline Sub-dimensions & $\begin{array}{l}\text { Feeling } \\
\text { Competent } \\
\text { Status }\end{array}$ & $\mathbf{N}$ & $\mathbf{X}$ & Sd & $\begin{array}{l}\text { Rank } \\
\text { Ave. }\end{array}$ & $x^{2}$ & p \\
\hline \multirow{3}{*}{ Educational Medium } & No & 72 & 13,44 & 1,807 & 78,62 & \multirow{4}{*}{1,002} & \multirow{4}{*}{,606 } \\
\hline & Partly & 78 & 13,79 & 1,523 & 86,03 & & \\
\hline & Yes & 14 & 13,57 & 1,785 & 82,82 & & \\
\hline \multirow{4}{*}{ Interpersonal Relations } & No & 72 & 37,76 & 4,650 & 81,70 & & \\
\hline & Partly & 78 & 37,67 & 4,772 & 81,90 & \multirow[t]{3}{*}{,381 } & \multirow[t]{2}{*}{,827 } \\
\hline & Yes & 14 & 38,79 & 5,508 & 89,96 & & \\
\hline & No & 72 & 36,88 & 4,990 & 79,18 & & \\
\hline \multirow[t]{3}{*}{ Work Life } & Partly & 78 & 37,37 & 5,213 & 84,79 & \multirow[t]{3}{*}{,651 } & \multirow[t]{3}{*}{,722 } \\
\hline & Yes & 14 & 37,50 & 5,681 & 86,79 & & \\
\hline & No & 72 & 9,99 & 2,255 & 81,69 & & \\
\hline \multirow[t]{3}{*}{ Family Life } & Partly & $7 \overline{8}$ & 9,94 & 2,565 & 81,87 & \multirow[t]{3}{*}{,404 } & \multirow[t]{3}{*}{,817 } \\
\hline & Yes & 14 & 10,43 & 2,027 & 90,14 & & \\
\hline & No & 72 & 27,96 & 3,966 & 76,85 & & \\
\hline \multirow[t]{3}{*}{ Personal Characteristics } & Partly & 78 & 28,79 & 3,736 & 86,71 & \multirow[t]{3}{*}{1,839} & \multirow[t]{3}{*}{,399 } \\
\hline & Yes & 14 & 28,93 & 3,812 & 88,11 & & \\
\hline & No & 72 & 48,85 & 6,062 & 78,81 & & \\
\hline \multirow[t]{3}{*}{ Competence - Independent Life } & Partly & $7 \overline{8}$ & 49,40 & 6,519 & 83,33 & \multirow[t]{3}{*}{1,749} & \multirow[t]{3}{*}{,417 } \\
\hline & Yes & 14 & 50,14 & 6,938 & 96,86 & & \\
\hline & No & 72 & 174,88 & 19,985 & 79,18 & & \\
\hline \multirow[t]{2}{*}{ Total Attitude Score } & Partly & 78 & 176,96 & 20,792 & 84,26 & \multirow[t]{2}{*}{,789 } & \multirow[t]{2}{*}{,674 } \\
\hline & Yes & 14 & 179,36 & 22,239 & 89,79 & & \\
\hline
\end{tabular}


Table 10. Comparison of the Attitudes of the Participants towards the Handicapped according to the Desire to Work in a School that Provides Education on Special Education (the Handicapped)

\begin{tabular}{|c|c|c|c|c|c|c|c|c|}
\hline Sub-dimensions & $\begin{array}{l}\text { Desire for } \\
\text { working }\end{array}$ & $\mathbf{N}$ & $x$ & Sd & $\begin{array}{l}\text { Rank } \\
\text { Ave. }\end{array}$ & Rank Total & $\mathbf{U}$ & $\mathbf{p}$ \\
\hline \multirow{2}{*}{ Educational Medium } & Yes & 73 & 13,49 & 1,827 & 80,35 & 5865,50 & \multirow{2}{*}{3164,5} & \multirow{2}{*}{,586 } \\
\hline & No & 91 & 13,73 & 1,542 & 84,23 & 7664,50 & & \\
\hline \multirow{2}{*}{$\begin{array}{l}\text { Interpersonal } \\
\text { Relations }\end{array}$} & Yes & 73 & 37,96 & 4,689 & 84,22 & 6148,00 & \multirow{2}{*}{3196,0} & \multirow{2}{*}{ 677 } \\
\hline & No & 91 & 37,68 & 4,844 & 81,12 & 7382,00 & & \\
\hline \multirow{2}{*}{ Work Life } & Yes & 73 & 36,77 & 5,453 & 79,25 & 5785,00 & \multirow{2}{*}{3084,0} & \multirow{2}{*}{,431 } \\
\hline & No & 91 & 37,48 & 4,863 & 85,11 & 7745,00 & & \\
\hline \multirow{2}{*}{ Family Life } & Yes & 73 & 10,03 & 2,522 & 83,18 & 6072,00 & \multirow{2}{*}{3272,0} & \multirow{2}{*}{,869 } \\
\hline & No & 91 & 9,98 & 2,275 & 81,96 & 7458,00 & & \\
\hline \multirow{2}{*}{$\begin{array}{l}\text { Personal } \\
\text { Characteristics }\end{array}$} & Yes & 73 & 28,33 & 3,823 & 81,19 & 5927,00 & \multirow{2}{*}{3226,0} & \multirow{2}{*}{,751 } \\
\hline & No & 91 & 28,53 & 3,883 & 83,55 & 7603,00 & & \\
\hline \multirow{2}{*}{$\begin{array}{l}\text { Competence - } \\
\text { Independent Life }\end{array}$} & Yes & 73 & 48,78 & 6,700 & 79,34 & 5792,00 & \multirow{2}{*}{3091,0} & \multirow{2}{*}{,444 } \\
\hline & No & 91 & 49,57 & 6,032 & 85,03 & 7738,00 & & \\
\hline \multirow{2}{*}{ Total Attitude Score } & Yes & 73 & 175,36 & 21,396 & 80,79 & 5897,50 & \multirow{2}{*}{3196,5} & \multirow{2}{*}{,679 } \\
\hline & No & 91 & 176,97 & 19,810 & 83,87 & 7632,50 & & \\
\hline
\end{tabular}

required by the vast majority of teachers were often summarized in the "general information on students with special needs” sub-dimension. In the study, it was also stated that the department graduated by the teachers and the seniority levels have effects on the in-service needs of special education. In this study in which the in-service training requirements for special training on the field were determined, it was found that there are differences between the years of service of the teachers and the service level variables. In another study conducted with the same scale that we used in our study, a study was conducted on university students who were educated in the child development department by using a single group and pre-test-post-test control groups without a control group by using the experimental design. In the study conducted in Namık Kemal University, SHMYO Child Development Department, the purpose was to determine the effectiveness of a training program for the handicapped students on the attitudes of the students towards the handicapped.

In order to determine the effect of the training program on the attitudes of the students towards the disabled individuals, the attitude scores obtained by OYTO in the pre-test and post-test sessions of the participants were compared in the scope of the total scale and all other sub-dimensions. The increase in the scores obtained from the total and each subscale of the SCM is interpreted as the increase in the attitude of the students towards the handicapped individuals in the positive direction. When the results obtained from the analysis based on the total of the scale are examined, it may be said that the training program applied has positive effects on the attitudes of the participants towards the handicapped in general terms [14]. It is believed that students with integration students in their classes will develop positive attitudes towards their handicapped classmates [15]. In a study of teacher attitudes, it was revealed that class teachers were in a negative attitude towards working in classrooms where mentally handicapped children were present, and that they had to focus their efforts to change their attitudes positively [16]. The information program has a positive effect on the attitudes of normal classroom teachers to the hearing-impaired children in the integration environment [17]. In a similar research, it has been examined whether the integration process has been carried out in terms of the attitudes towards the integration of classroom teacher candidates and it has been found that a course related to integration affects attitudes positively [18]. Teachers' effectiveness in changing their attitudes towards cohesion to being informed about the integration of special needs children was examined and positive changes were found in attitudes [19]. In a research conducted, the opinions of the teachers who applied the mainstreaming were examined. It has been found out that teachers do not have enough knowledge to integrate [20]. In another research, it has been determined that the special education counselling and integration course has a positive effect on the attitudes of prospective teachers to the integration education [21].

In a study conducted by Ağbuğa and Gürsel [3], the effectiveness was evaluated for reading a panel text about family matters and social experiences by $1^{\text {st }}$ and $2^{\text {nd }}$ grades of the School of Physical Education and Sports in changing their attitudes towards the disabled. The panel text was read after the pre-test by $1^{\text {st }}$ and $2^{\text {nd }}$ year students of Ankara University BESYO who did not take any courses related to disabilities. The students were then given the post-test. There was a significant and positive change in the attitudes of these students measured by the YEKYT Scale adapted to Turkish for those affected by physical disabilities. According to the result of this study, informing may be effective in changing the attitudes of university students.

The basic aim of the present study of ours was to examine the education level -if any- of the physical education teachers on special education and determine their 
awareness levels on special awareness. When we look at the descriptive statistics in our study, it is determined that $37.8 \%$ of the participants were female, and $62.2 \%$ were male. There was no statistically significant difference in the attitudes towards the handicapped according to Gender and Age. Female and Male participants were found to have very close sub-dimensions and total attitude scores.

When we examined the level of awareness of the participants according to the educational status of the participants, it was seen that there were no doctorate level participants and the Post-graduate and Undergraduate students did not differ at a statistically significant level in both sub-dimensions and in terms of general awareness ( $>0,05$ ). 92.7\% of the participants were graduated from departments that were close to physical education, 5.5\% were from classroom teachers' departments and 1.8\% were graduated from sports administration, recreation and coaching departments. We can see that there is no statistically significant difference when we look at the study results according to the departments where the participants graduated ( $>0,05)$. However, it is seen that the total attitude scores of classroom teacher graduates are higher. We may claim that the reason for this is the existence of compulsory courses on special education in classroom teaching departments.

When we examined the level of awareness on disabilities according to whether or not participants were educated about special education, it was determined that they did not differ at a statistically significant level in both sub-dimensions and general awareness levels ( $p>0,05)$. This conclusion is an expected finding in our study and is an opposite finding for similar studies because education or information on a topic affect the attitudes. We can evaluate it as one of the limitations of the present study; and also, it is possible that the experiences of the participants might have been influential on this result.

When we look at the levels of awareness about the handicapped according to the working status in a special education school, it is seen that they are not statistically different in both sub-dimensions and general awareness levels $(p>0,05)$. Although there is no statistically significant difference, it is seen that the average attitude scores of the participants who are currently working in a special education institution are higher than those who do not work in the private education institution at all.

One of the interesting findings of our study is that the level of awareness on the disabilities did not differ at a statistically significant level in the sub-dimensions and general awareness levels, depending on whether participants found themselves adequately qualified for special education $(p>0,05)$. But the important point here is that $91.4 \%$ of the participants answered as "Partly" and "No" to the question "Do you think that you are qualified for special education?”. Only $8.6 \%$ of respondents feel competent about special education. When we think that all of the participants have the qualifications to be assigned to special education institutions, the number of the teachers who feel competent attracts attention as being very few.

Finally, when we look at the levels of awareness on disabilities according to whether or not the teachers want to work in a school that provides special education, it is seen that they do not differ statistically in both subdimensions and general awareness level ( $\mathrm{p}>0,05)$. However, we see that $55.4 \%$ of the participants do not want to work in a school that provides special education. As one of the reasons, it is possible to say that they see themselves as incompetent in this regard.

\section{Conclusion}

As a result, in-service training comes to the mind first as a remedy in this respect when it is considered that branch teachers who are assigned to special education schools and job training centres do not have a specific education because there were no Undergraduate departments in their university years and they only received a few-credit special education courses in their departments. However, the quality of in-service training and the qualifications of those who provide in-service training do not appear to be sufficient to fill this gap. For this reason, it seems necessary to open special education teaching departments as specific to the area that will provide education at undergraduate level. The first field of study that may be opened specifically for this field is the Special Education-Physical Education Teaching. An education at the undergraduate level in this area will provide that teachers are directed to this field; and it will also increase the quality of education.

\section{Recommendations}

It is known that branch teachers who are assigned to special education and business practice schools are appointed to these schools without any qualifications for special education. It is clear that there will be negative consequences of this. For this reason, if the teachers to be assigned to these schools are inadequate, trainings related to the subject can be provided (in-service training, etc.).

Informative work such as in-service training may not be sufficient in an area where such expertise is required. Therefore, special education teaching departments can be opened for departments offering undergraduate education such as "Special Education-Physical Education Teacher".

Special education groups are subject to many different classifications in their own group. Even students with special education needs within the same classification can have different characteristics. This requires different expertise for each special education group that is categorized. Based on this, physical education teachers can be given specialist training for different special education groups.

\section{Financing}

No financial support was received for the research.

This paper is presented at "World Congress of Sport Sciences Researches” 23-26 November 2017, Manisa / Turkey

\section{Conflict of interests}

The authors declare that there is no conflict of interests. 
1. Aydın O. Davranıs bilimine giriș-4. [Introduction to behavioral science-4]. Anadolu University Open Education Faculty Publications; 1985. (In Turkish)

2. Özyürek M. Effects of live, audio and print presentation of a discussion about physical disabilities on attitude motivation toward disable person in Turke. [PhD thesis]. University of Northern Colorado; 1977.

3. Akbuğa B, Gürsel F. Bilgilendirme yoluyla beden eğitimi spor yüksekokulu öğrencilerinin engelli bireye yönelik değişen tutumları. [Physical education through informing changing attitudes of students of sports college for disabled persons]. Spormetre Beden Eğitimi Ve Spor Bilimleri Dergisi. 2006;1:5-8. (In Turkish)

4. Aktaş $\mathrm{C}$, Küçüker $\mathrm{S}$. Bilişsel-duyuşsal odaklı bir programın ilköğretim öğrencilerinin fiziksel engelli yaşıtlarına yönelik sosyal kabul düzeylerine etkisinin incelenmesi. [An analysis of the effect of a cognitive-affective focused program on the level of social acceptance of physically handicapped students of primary school students]. Ankara Üniversitesi Ë̆itim Bilimleri Fakültesi, Özel Eğitim Dergisi. 2001;3(2): 15-25. (In Turkish)

5. Hogg MA, Vaughan GM. Sosyal psikoloji. [Social psychology]. Ankara: Utopya; 2006. (In Turkish)

6. Taylor SE, Peplau LA, Sears DO. Sosyal psikoloji. [Social psychology]. Ankara: İmge; 2007. (In Turkish)

7. Riddel S, Weedon E. Changing legislation and its effects on inclusive and special education: Scotland. British Journal of Special Education. 2014; 41( 4): 363- 381.

8. Griffin CC, Kilgore KL, Winn JA, Wilborn AO, Hou W, Garvan CW. First-year special educators the influence of school and classroom context factors on their accomplishments and problems. Teacher Education and Special Education. 2009;32(1); 45-63.

9. Brownell MT, Hirsch E, Seo S. Meeting the demand for highly qualified special education teachers during severe shortages: what should policymakers consider? The Journal of Special Education. 2004; 38 (1); 56-61.

10.Ngang TK. A comparative study on teacher leadership in special education classroom between China and Malaysia. Procedia - Social and Behavioural Sciences. 2012; 31; 231 $-235$.

11.T.C. Başbakanlık Özürlüler İdaresi Başkanlığı. Toplum özürlülüğü nasıl anlıyor araştırması. [How to understand community disability research]. 2009; 49-57:36-38. (In Turkish)

12.Gözün Ö, Yıkmış A. Öğretmen adaylarının kaynaştırma konusunda bilgilendirilmelerinin kaynaştırmaya yönelik tutumlarının değişimindeki etkililiğii. [The effectiveness of teacher candidates' knowledge of inclusion in changing attitudes towards cohesion]. National Special Education Congress. 2003; 13:120-136. (In Turkish)

13.Sazak EP. Kaynaştırma sınıfı öğretmenlerinin özel eğitimle ilgili hizmet içi eğitim gereksinimlerinin belirlenmesi.[
Determination of in-service training needs of mainstreaming teachers for special education]. National Special Education Congress, 2013;23: 120-125. (In Turkish).

14.Şahin F, Güldenoğlu B. engelliler konusunda verilen eğitim programının engellilere yönelik tutumlar üzerindeki etkisi. [ Impact of the training program on disabilities on attitudes towards disability]. Amasya University, Journal of Education Faculty, 2013; 2(1): 214-239. (In Turkish)

15.Batu S, İftar GK, Uzuner Y. Özel gereksinimli öğrencilerin kaynaştırıldiğ $\mathrm{l}$ bir kız meslek lisesindeki ögretmenlerin kaynaştırmaya ilişkin görüş ve önerileri. [Opinion and recommendation of the integration of teachers in a girl's vocational school where special needs students are integrated]. Ankara University, Faculty of Educational Sciences, Özel Eğitim Journal, 2004; 5(2): 98-102. (In Turkish)

16.Diken H, Sucuoğlu B. Sınıfında zihin engelli çocuk bulunan ve bulunmayan sınıf öğretmenlerinin zihin engelli çocukların kaynaştırılmasına yönelik tutumlarının karşılaştırılması. [Comparison of classroom teachers with and without mentally handicapped children's attitudes towards the integration of mentally handicapped children in their class]. Special Education Journal. 1999; 2(3): 25-39. (In Turkish)

17.Kayaoğlu H. Bilgilendirme programının normal sinıf ögretmenlerinin kaynaştırma ortamındaki işitme engelli çocuklara yönelik tutumlarına etkisi. [Impact of the information program on the attitudes of normal classroom teachers to the hearing-impaired children in the integration environment]. [M.Sc thesis]. Ankara: Ankara University; 1999. (In Turkish)

18.A, Zerey Z, Töret G. Sınıf öğretmeni adaylarının kaynaştırmaya yönelik tutumlarının incelenmesi. [Examining the attitudes of classroom teacher candidates towards integration]. Journal of Special Education. 2004; 5(1): 2333. (In Turkish)

19.Şahbaz Ü. Öğretmenlerin özel gereksinimli çocukların kaynaştırılması konusunda bilgilendirilmelerinin kaynaştırmaya ilişsin tutumlarının değişmesindeki etkililiği. [Effectiveness of teachers' attitudes towards cohesion to be informed about the integration of special needs children]. [M.Sc thesis]. Abant Izzet Baysal University; 1997. (In Turkish)

20.Uysal A. Kaynaştırma uygulaması yapan öğretmenlerin kaynaştırmaya ilişskin görüşleri. [The opinions of the teachers who practice the mainstreaming]. National Special Education Congress. Ankara: Kök Publishing. 2003; 13: 121-135. (In Turkish)

21.Yıkmış A, Şahbaz Ü, Peker S. Özel eğitim danışmanlığ 1 ve kaynaştırma dersinin öğretmen adaylarının kaynaştırmaya yönelik tutumlarına etkisi. [The effect of special education counseling and integration course on teacher candidates' attitudes towards integration]. National Special Education Congress. Edirne. 1998; 8: 163-167. (In Turkish) 
Information about the authors:

Yaman Ç.; Assoc.Prof.Dr; http://orcid.org/0000-0002-0888-2154; cyaman@sakarya.edu.tr; Faculty of Sport Science, Recreation, Sakarya University; 54187 - Esentepe /Sakarya, Turkey.

Uluışık V. (Corresponding author); Phd Student; http://orcid.org/0000-0002-9460-7270; vedat151515@gmail.com; İnstitute of Educational Science, Physical Education and Sport Teaching, Sakarya University; 54300-Hendek/Sakarya, Turkey.

Hergüner G.; Assoc.Prof.Dr; http://orcid.org/0000-0002-3087-3684; herguner@sakarya.edu.tr; Faculty of Sport Science, Physical Education and Sport Teaching, Sakarya University; 54187 - Esentepe /Sakarya, Turkey.

Önal A.; Phd Student; http://orcid.org/0000-0003-3786-6679; aysedurukonal@hotmail.com; İnstitute of Educational Science, Physical Education and Sport Teaching, Sakarya University; 54300-Hendek/Sakarya, Turkey.

Cite this article as: Yaman Ç, Uluışık V, Hergüner G, Önal A. Examining the attitudes of physical education teachers towards special education (the handicapped). Physical education of students, 2018;22(4):2017-216. doi:10.15561/20755279.2018.0406

The electronic version of this article is the complete one and can be found online at: http://www.sportedu.org.ua/index.php/PES/issue/archive

This is an Open Access article distributed under the terms of the Creative Commons Attribution License, which permits unrestricted use, distribution, and reproduction in any medium, provided the original work is properly cited (http://creativecommons.org/licenses/by/4.0/deed.en).

Received: 11.06.2018

Accepted: 06.07.2018; Published: 23.08.2018 